\title{
WAVE PROPAGATION CHARACTERISTICS IN FUNCTIONALLY GRADED DOUBLE-BEAMS
}

\author{
Fatih Karacam', Metin Aydogdu' \\ 1 Trakya University, Faculty of Engineering, Department of Mechanical Engineering, Edirne, TURKEY, e-mail: \\ fatihkar@trakya.edu.tr, metina@trakya.edu.tr
}

Received: 2017.05.15

Accepted: 2017.08.01

Published: 2017.09.03

\begin{abstract}
The wave propagation characteristics of functionally graded (FG) double-beams are investigated by use of Euler-Bernoulli beam theory. Two beams are connected by a Winkler foundation. The wave propagation characteristics like frequency, phase and group velocities are obtained for different wave numbers and material properties. Four frequencies are obtained for functionally graded double-beam system. It is obtained that flexural and axial waves are coupled for FG double-beams.
\end{abstract}

Keywords: Functionally graded material, double-beam, wave propagation.

\section{INTRODUCTION}

Since functionally graded materials (FGM) have high strength, temperature resistance, corrosion resistance and toughness, they are widely used in structures such as beams and plates. In functionally graded materials, properties are continuously changing in one or more directions. Due to the continuous stress distribution along a specific direction in the structures, they have many applications in industrial and engineering fields. They have a steady micro-structure characteristics changing from one material to another to obtain the optimum desired parameters in the structure. Double-beam systems that consist of two parallel beams are connected by an elastic layer and have many usages in mechanical, civil and aeronautical applications such as doublebeam cranes, double-beam spectrometers, floating-slab tracks, railway tracks, pipelines, bridge spans and aircraft wing spars. Due to high temperature resistance and continuous stress distribution, studies in recent years have focused on the problems of structures which are constructed of functionally graded materials. Abu-Hilal [1] studied the dynamic response of a double-beam system consisting two elastic homogenous isotropic
Euler-Bernoulli beams and obtained the dynamic deflections for different values of speed, damping ratio and stiffness parameters. Arefi and Zenkour [2] used Timoshenko beam model for a functionally graded magneto-electric nano-beams resting on Visco-Pasternak foundation and studied the effect of material inhomogeneities and wave number on the wave propagation of the nano-beam in different modes. Ariaei et al [3] investigated the dynamic response of an elastically connected multiple-beam system by using Timoshenko beam theory and obtained the midpoint deflections for different values of moving load velocity and the stiffness of elastic connections. Aydogdu and Taskin [4] investigated free vibration behavior of simply supported functionally graded beams by use of classical, parabolic and exponential shear deformation beam theories and obtained the free vibration frequencies for different materials and length to thickness ratios. Chen et al [5] proposed a mixed method that combines the state space method and the differential quadrature method for the bending and free vibration analysis of thick beams resting on a Pasternak elastic foundation. They obtained the mid-span deflections for different boundary conditions and investigated the effects of Poisson's ratio on the fundamental fre- 
quencies. Deng et al [6] established the dynamic stiffness matrix of a double-functionally graded Timoshenko beam system on Winkler-Pasternak elastic foundation under axial loading and obtained the exact natural frequency and buckling load. De Rosa and Maurizi [7] investigated the exact free vibration frequencies of Euler beam on two-parameter elastic soil with flexible ends under a concentrated mass acting along the span. They normalized the equation of motion by introducing two different reference frames and simplified the frequency equation as much as possible. Hussein and Hunt [8] discussed modelling of floating-slab tracks on rigid foundations. They used two continuous resilient layers such as upper and lower Euler-Bernoulli beams in the model to obtain the displacements that are used to calculate the cut on frequencies and critical velocities of the track. $\mathrm{Li}$ et al [9] developed an analytical model of smallscaled functionally graded beams for the flexural wave propagation analysis and investigated the dispersion relation between the phase velocity and wave number. Shamalta and Metrikine [11] investigated the steady-state dynamic response of an embedded railway track by use of a model composing of two Euler-Bernoulli beams, a Kirchoff plate, two continuous visco-elastic elements that connect the beams and plate and 2-D elastic foundation. $\mathrm{Vu}$ et al [13] presented an exact solution for solving the vibration of a double-beam system consisting of a main beam with an applied force and an auxiliary beam with a distributed spring and dashpot between two beams and subjected it to a harmonic excitation. Ying et al [15]

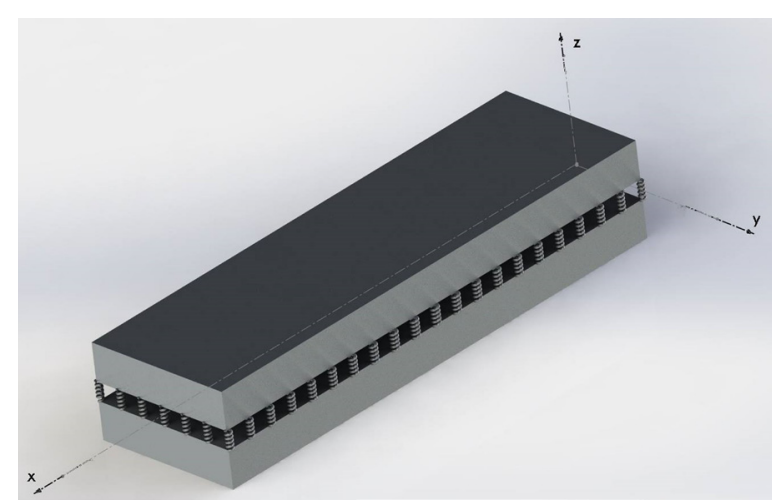

Fig. 1. Double-beam geometry and coordinate system

presented a two-dimensional theory of elasticity in the bending and free vibration analysis of functionally graded beams resting on a WinklerPasternak elastic foundation. They investigated the effects of several parameters such as gradient index, aspect ratios and foundation parameters on mechanical behavior of FGM beams. Zhang et al [16] investigated the flexural wave propagation of piezo electric functionally graded nano-beams with thermal and surface effects. According to the author's knowledge, wave propagation characteristics of functionally graded double-beam haven't been investigated. In this study, the wave propagation characteristics of functionally graded double-beams are developed on the basis of Euler-Bernoulli beam theory. By use of the analytical model, the wave propagation characteristics such as frequency, phase and group velocities are obtained and the variation of these parameters with respect to wave numbers are illustrated.

\section{THEORY AND FORMULATION}

In the study, double-beam system is assumed to be constructed of two parallel beams with a length of $L$, unit width, thickness of $h$ and Winkler elastic layer between them. Figure 1 represents the doublebeam geometry and coordinate system which is placed in the mid-plane.

Upon denoting $U, V$ and $W$ as the displacement fields in $x$-, $y$ - and $z$-axis respectively and assuming that the displacement along $y$-axis is zero, the displacement fields are assumed in parallel with the general shear deformation shell theory introduced by Soldatos and Timarci [12],

$$
\begin{gathered}
U(x, z ; t)=u(x ; t)-z w_{, x}+\emptyset(z) u_{1}(x ; t) \\
V(x, z ; t)=0 \\
W(x, z ; t)=w(x ; t)
\end{gathered}
$$

where: $u, u_{1}$ and $w$ are three unknown displacement functions of the middle surface of the beam and subscript "," denotes the differentiation with relevant axis. $\varnothing(z)$ represents the shape function that determines the distribution of the transverse shear stress and strain. Moreover, the choice of different shape functions will lead to different beam theories. The displacement fields yield the following kinematic relations: 


$$
\varepsilon_{x}=u_{, x}-z w_{, x x}+\emptyset(z) u_{1, x} \quad \gamma_{x z}=\emptyset^{\prime}(z) u_{1}
$$

Here, prime denotes the differentiation with respect to $z$-axis. The state of stresses for each $\mathrm{n}^{\text {th }}$ layer is given by generalized Hooke's law as follows:

$$
\left[\begin{array}{c}
\sigma_{x}^{(n)} \\
\tau_{x z}^{(n)}
\end{array}\right]=\left[\begin{array}{cc}
Q_{11}^{(n)} & 0 \\
0 & Q_{55}^{(n)}
\end{array}\right]\left[\begin{array}{c}
\varepsilon_{x} \\
\gamma_{x z}
\end{array}\right]
$$

$Q_{i j}$ is the transformed reduced stiffness depending on the mechanical properties of material such as elasticity modulus $E(z)$ and Poisson's ratio $v$ and given in the following form:

$$
Q_{11}=\frac{E(z)}{1-v^{2}} \quad Q_{55}=\frac{E(z)}{2\left(1+v^{2}\right)}
$$

$v$ is assumed as a constant in the present study since the constituents of functionally graded beam are approximately identical. Elasticity modulus $E(z)$, is assumed to be changing through the thickness of the beam according to power law form which is introduced by Wakashima [14] and given as follows:

$$
E(z)=\left(E_{T(i)}-E_{B(i)}\right)\left(\frac{z}{h}+\frac{1}{2}\right)^{m}+E_{B(i)} \quad(i=1,2)
$$

$E_{T(i)}$ and $E_{B(i)}$ are the elasticity modulus of beam at top and bottom surfaces respectively and $m$ indicates the power-law index. Top surface of the beam is assumed to be constructed of ceramics whereas the bottom surface is aluminum. In Figure 2, the variation of the elasticity modulus with respect to the beam thickness is presented for different power-law indices.

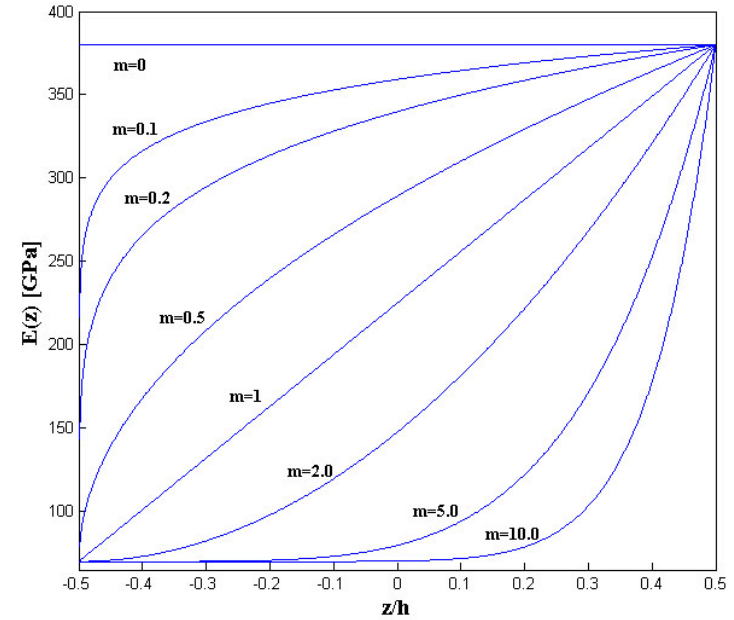

Fig. 2. The variation of elasticity modulus with beam thickness

In the study, shape function is chosen as 0 so as to correspond to Euler-Bernoulli beam theory. When the stress-strain relations are substituted into the force and moment definitions:

$$
N_{x}=\int_{-h / 2}^{h / 2} \sigma_{x} d z \quad M_{x}=\int_{-h / 2}^{h / 2} \sigma_{x} z d z
$$

the following constitutive equations are obtained as follows:

$$
\left[\begin{array}{l}
N_{x} \\
M_{x}
\end{array}\right]=\left[\begin{array}{ll}
A_{11} & B_{11} \\
B_{11} & D_{11}
\end{array}\right]\left[\begin{array}{c}
u_{, x} \\
-w_{, x x}
\end{array}\right]
$$

where: $A_{i j}, B_{i j}$ and $D_{i j}$ denote the extensional, coupling and bending stiffnesses respectively. The extensional, coupling and bending stiffnesses are defined in the following way:

$$
\begin{gathered}
A_{11}=\int_{-h / 2}^{h / 2} Q_{11} d z \\
B_{11}=\int_{-h / 2}^{h / 2} Q_{11} z d z \\
D_{11}=\int_{-h / 2}^{h / 2} Q_{11} z^{2} d z
\end{gathered}
$$

The governing equations of the beam can be obtained variationally by use of Hamilton's principle as follows:

$$
\begin{gathered}
N_{x, x}=\left(\rho_{0} u-\rho_{1} w_{, x}\right)_{, t t} \\
M_{x, x x}=\left(\rho_{1} u_{, x}+\rho_{0} w-\rho_{2} w_{, x x}\right)_{, t t}
\end{gathered}
$$

where: the subscript ",tt" denotes the derivation with respect to time. $\rho_{\mathrm{i}}$ is the density function of the beam and defined in the following form:

$$
\rho_{i}=\int_{-h / 2}^{h / 2} \rho z^{i} d z, \quad(i=0)
$$


By neglecting the rotary inertias, the equations of motion of a double-beam system with an elastic layer, can be defined as (Filiz and Aydogdu [10]),

$$
\begin{gathered}
A_{11}^{(1)} u_{, x x}^{(1)}-B_{11}^{(1)} w_{, x x x}^{(1)}=\rho_{0}^{(1)} u_{, t t}^{(1)} \\
B_{11}^{(1)} u_{, x x x}^{(1)}-D_{11}^{(1)} w_{, x x x x}^{(1)}=\rho_{0}^{(1)} w_{, t t}^{(1)}-c\left(w^{(2)}-w^{(1)}\right) \\
A_{11}^{(2)} u_{, x x}^{(2)}-B_{11}^{(2)} w_{, x x x}^{(2)}=\rho_{0}^{(2)} u_{, t t}^{(2)} \\
B_{11}^{(2)} u_{, x x x}^{(2)}-D_{11}^{(2)} w_{, x x x x}^{(2)}=\rho_{0}^{(2)} w_{, t t}^{(2)}+c\left(w^{(2)}-w^{(1)}\right)
\end{gathered}
$$

Superscripts (1) and (2) represent the first and second beam, respectively and $c$ denotes the stiffness of elastic medium. For the wave propagation analysis, the displacement functions are considered as:

$$
u^{j}=A^{j} e^{i(\omega t-k x)} \quad(j=1,2) \quad w^{j}=B^{j} e^{i(\omega t-k x)} \quad(j=1,2)
$$

$\mathrm{A}^{\mathrm{j}}$ and $\mathrm{B}^{\mathrm{j}}$ are the amplitudes of wave motion, $k$ is the wave number and $\omega$ is the frequency. The equations of motion can be rewritten by the use of displacement functions depending on frequencies and wave number as,

$$
\begin{gathered}
A^{(1)}\left(A_{11}^{(1)} k^{2}-\rho_{0}^{(1)} \omega^{2}\right)+B^{(1)}\left(B_{11}^{(1)} i k^{3}\right)=0 \\
A^{(1)}\left(-B_{11}^{(1)} i k^{3}\right)+B^{(1)}\left(D_{11}^{(1)} k^{4}+c-\rho_{0}^{(1)} \omega^{2}\right)+B^{(2)}(-c)=0 \\
A^{(2)}\left(A_{11}^{(2)} k^{2}-\rho_{0}^{(2)} \omega^{2}\right)+B^{(2)}\left(B_{11}^{(2)} i k^{3}\right)=0 \\
B^{(1)}(-c)+A^{(2)}\left(-B_{11}^{(2)} i k^{3}\right)+B^{(2)}\left(D_{11}^{(2)} k^{4}+c-\rho_{0}^{(2)} \omega^{2}\right)=0
\end{gathered}
$$

The determinant of the coefficient matrix of homogeneous system of equations obtained above will give dispersion relations. Thus, the problem may be considered as an eigenvalue problem and in order to solve an eigenvalue problem computationally, the equations can be written in a matrix form as follows:

\begin{tabular}{|c|c|c|c|c|c|}
\hline \multirow{2}{*}{ Case } & & \multicolumn{2}{|c|}{$\mathrm{E}$ [GPa] } & \multirow{2}{*}{$\rho\left[\mathrm{kg} / \mathrm{m}^{3}\right]$} & \multirow{2}{*}{$\mathrm{m}$} \\
\hline & & $\mathrm{E}_{\mathrm{T}}$ & $\mathrm{E}_{\mathrm{B}}$ & & \\
\hline \multirow{2}{*}{1} & Beam 1 & 70 & 70 & 2770 & 0 \\
\hline & Beam 2 & 70 & 70 & 2770 & 0 \\
\hline \multirow{2}{*}{2} & Beam 1 & 380 & 70 & 2770 & 1 \\
\hline & Beam 2 & 380 & 70 & 3700 & 1 \\
\hline \multirow[b]{2}{*}{3} & Beam 1 & 380 & 70 & 2770 & 1 \\
\hline & Beam 2 & 380 & 70 & 3700 & 2 \\
\hline
\end{tabular}

$$
\left[\begin{array}{cccc}
\left(A_{11}^{(1)} k^{2}-\rho_{0}^{(1)} \omega^{2}\right) & \left(B_{11}^{(1)} i k^{3}\right) & 0 & 0 \\
\left(-B_{11}^{(1)} i k^{3}\right) & \left(D_{11}^{(1)} k^{4}+c-\rho_{0}^{(1)} \omega^{2}\right) & 0 & (-c) \\
0 & 0 & \left(A_{11}^{(2)} k^{2}-\rho_{0}^{(2)} \omega^{2}\right) & \left(B_{11}^{(2)} i k^{3}\right) \\
0 & (-c) & \left(-B_{11}^{(1)} i k^{3}\right) & \left(D_{11}^{(2)} k^{4}+c-\rho_{0}^{(2)} \omega^{2}\right)
\end{array}\right]\left\{\begin{array}{l}
A^{(1)} \\
B^{(1)} \\
A^{(2)} \\
B^{(2)}
\end{array}\right\}=0
$$

\section{RESULTS AND DISCUSSION}

For the doublty modulus, density and power-law indices. In Table 1, the material properties and power-law indices are presented for three different cases. In the first case, the double-beam is assumed to be constructed of the same materials, thus, the same material properties are used. In order to do this, power-law indices are chosen as 0 so as to correspond to an isotropic double-beam. In the second

Table 1. The material properties and power-law indices of double-beams for three different cases 
and third cases, the double-beam is assumed to be constructed of two beams with different material properties and power-law indices.

In Figure 3-4-5, the variation of frequency $(\mathrm{rad} / \mathrm{s})$, phase and group velocities $(\mathrm{m} / \mathrm{s})$ with wave numbers are presented respectively and four dispersion relations are obtained. Higher two of these roots are for flexural waves and lower two curves correspond to axial waves for this isotropic case. Dispersion curves of flexural (axial) cases are approximately coincident. Difference between flexural and axial dispersion curves increases as $k$ wave number increases. In Figure 6-7-8 and 9-10-11, wave dispersion curves are given for FG double-beams. It should be noted that curves for flexural and axial wave, are not coincident for this case. This is due to $B_{i j}$ coupling terms, nonzero $B_{i j}$ terms lead to the coupling between flexural and axial waves as it can be seen from (Eq. 11-13). Difference between flexural and

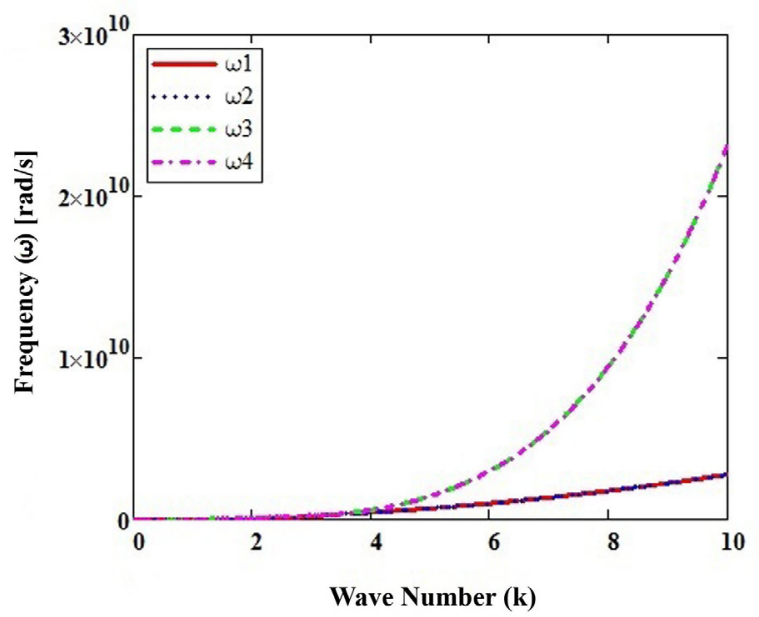

Fig. 3. The variation of frequency with wave number for Case 1

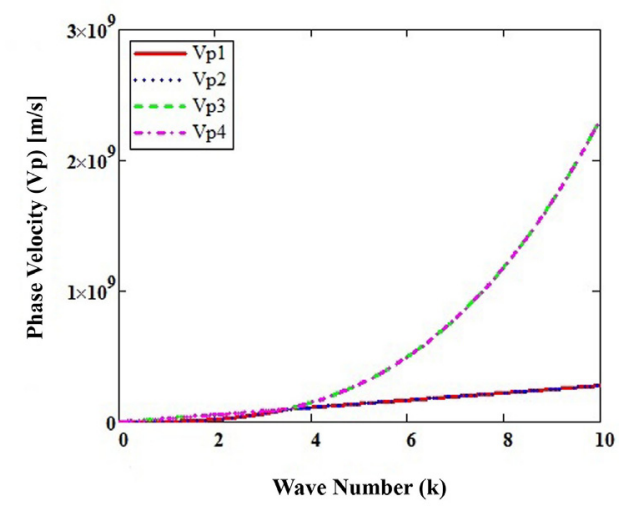

Fig. 4. The variation of phase velocity with wave number for Case 1 axial dispersion curves increases with increasing wave number. Similar behavior is observed for phase and group velocities. It is interesting to note that flexural dispersion curves get further whereas axial dispersion curves get closer with increasing wave number $k$ for Fig. 9-10-11.

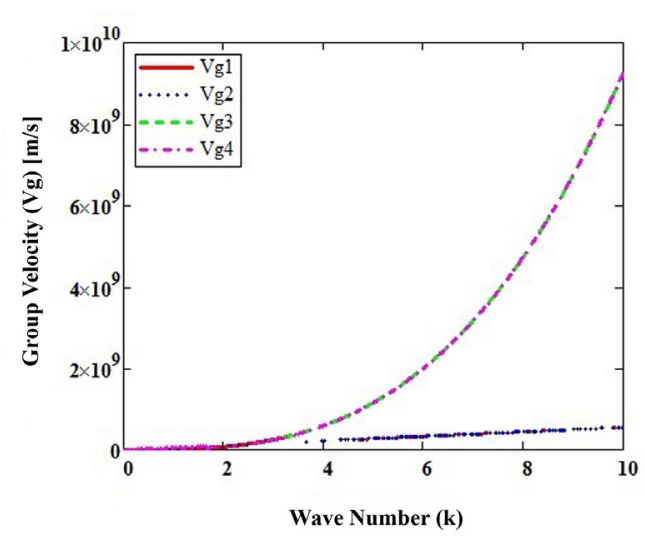

Fig. 5. The variation of group velocity with wave number for Case 1

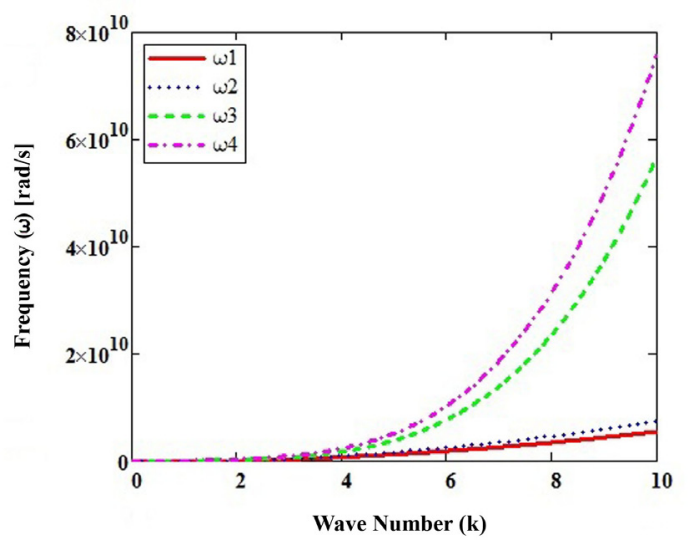

Fig. 6. The variation of frequency with wave number for Case 2

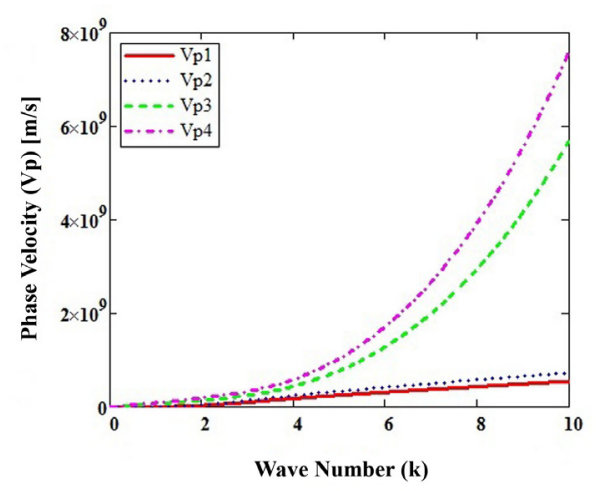

Fig. 7. The variation of phase velocity with wave number for Case 2 


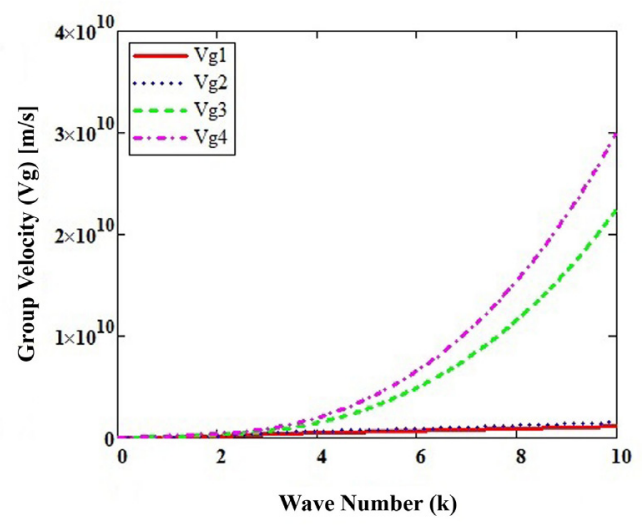

Fig. 8. The variation of group velocity with wave number for Case 2

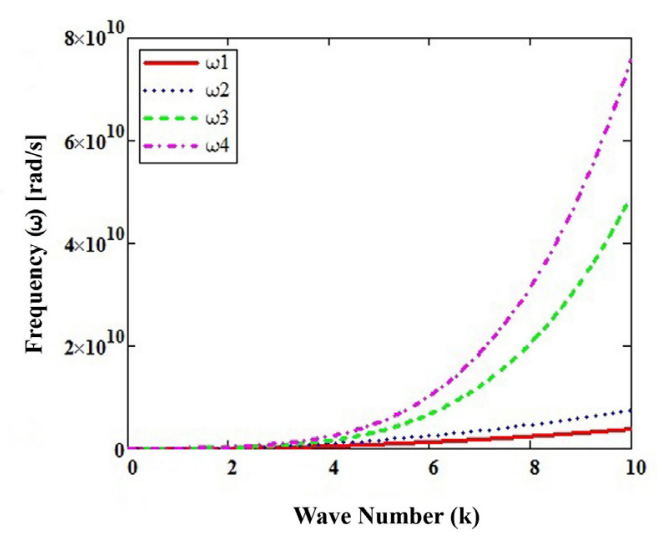

Fig. 9. The variation of frequency with wave number for Case 3

\section{CONCLUSIONS}

In this study, wave propagation analysis of functionally graded double-beam with Winkler elastic foundation is performed. Wave propagation characteristics like frequency, phase and group velocities are obtained for different wave numbers and three different cases. Initially, an isotropic double-beam system is considered. Four frequencies are obtained and the isotropic curves are coincident as expected. In the second and third cases, FG double-beams are considered for different material properties and power-law indices. A coupling between flexural and axial waves is observed. This study can be extended by use of a higher order beam theory for different parameters.

\section{REFERENCES}

1. Abu-Hilal M.: Dynamic response of a double Euler-Bernoulli beam due to a moving con-

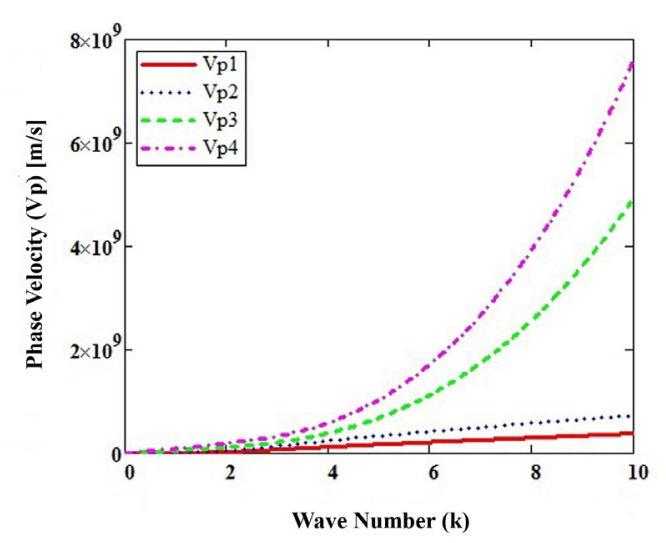

Fig. 10. The variation of phase velocity with wave number for Case 3

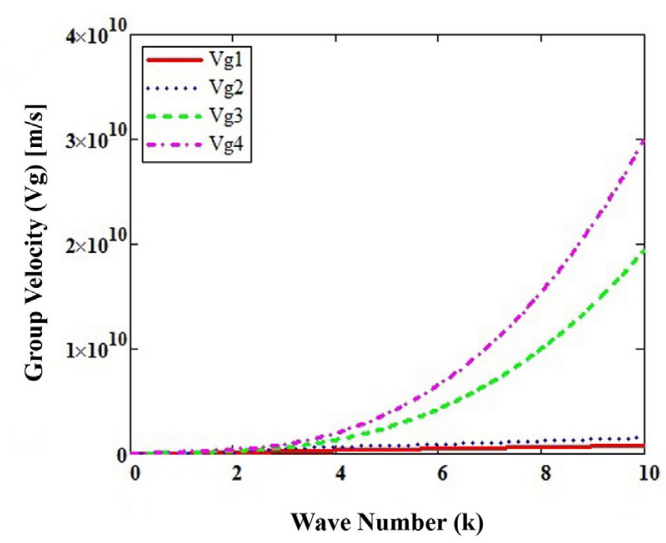

Fig. 11. The variation of group velocity with wave number for Case 3

stant load. Journal of Sound and Vibration, 297, 2006, 477-491.

2. Arefi M., Zenkour A.M.: Wave propagation analysis of a functionally graded magneto-electro-elastic nanobeam rest on Visco-Pasternak foundation. Mechanics Research Communications, 79, 2017, 51-62.

3. Ariaei A., Ziaei-Rad S., Ghayour M.: Transverse vibration of a multiple-Timoshenko beam system with intermediate elastic connections due to a moving load. Archive of Applied Mechanics, 81, 2011, 263-281.

4. Aydogdu M., Taskin V.: Free vibration analysis of functionally graded beams with simply supported edges. Materials and Design, 28, 2007, 1651-1656.

5. Chen W.Q., Lü C.F., Bian Z.G.: A mixed method for bending and free vibration of beams resting on a Pasternak elastic foundation. Applied Mathematical Modelling, 28, 2004, 877-890.

6. Deng H., Chen K.D., Cheng W., Zhao S.G.: Vibration and buckling analysis of double-functionally graded Timoshenko beam system on Winkler-Pasternak elastic foundation. Composite Structures, 160, 2017, 152-168. 
7. De Rosa M.A., Maurizi M.J.: The influence of concentrated masses and Pasternak soil on the free vibrations of Euler beams-exact solution. Journal of Sound and Vibration, 212, 4, 1998, 573-581.

8. Hussein M.F.M., Hunt H.E.M.: Modelling of floating-slab tracks with continuous slabs under oscillating moving loads. Journal of Sound and Vibration, 297, 2006, 37-54.

9. Li L., Hu Y., Ling L.: Flexural wave propagation in small-scaled functionally graded beams via a nonlocal strain gradient theory. Composite Structures, 133, 2015, 1079-1092.

10. Seckin F., Aydogdu M.: Wave propagation analysis of embedded (coupled) functionally graded nanotubes conveying fluid. Composite Structures, 132, 2015, 1260-1273.

11. Shamalta M., Metrikine A.V.: Analytical study of the dynamic response of an embedded railway track to a moving load. Archive of Applied Mechanics, 73, 2003, 131-146.
12. Soldatos KP, Timarci T. A unified formulation of laminated composite, shear deformable, five degrees of freedom cylindrical shell theories. Composite Structures, 25, 1993, 165-71.

13. Vu H.V., Ordonez A.M., Karnopp B.H.: Vibration of a double-beam system. Journal of Sound and Vibration, 229, 4, 2000, 807-822.

14. Wakashima K, Hirano T, Niino M. Space applications of advanced structural materials. ESA SP303:97; 1990.

15. Ying J., Lü C.F., Chen W.Q.: Two-dimensional elasticity solutions for functionally graded beams resting on elastic foundations. Composite Structures, 84, 2008, 209-219.

16.Zhang Y.W., Chen J., Zeng W., Teng Y.Y., Fang B., Zang J.: Surface and thermal effects of the flexural wave propagation of piezoelectric functionally graded nanobeam using nonlocal elasticity. Computational Materials Science, 97, 2015, 222-226. 